\title{
Design and Implementation of Polyphase based Subband Adaptive Structure for Noise Cancellation
}

\author{
Pratik Ghotkar ${ }^{1}$, Mrinal Bachute ${ }^{2}$, R. D. Kharadkar ${ }^{3}$ \\ ${ }^{I}$ (Dept. of E\&TC, GHRIET, University of Pune, India) \\ ${ }^{2}$ (Dept. of E\&TC, GHRIET, University of Pune, India) \\ 3 (Dept. of E\&TC, GHRIET, University of Pune, India)
}

\begin{abstract}
With the tremendous growth in the Digital Signal processing technology, there are many techniques available to remove noise from the speech signals which is used in the speech processing. Widely used LMS algorithm is modified with much advancement but still there are many limitations are introducing. This paper consist of a new approach i.e. subband adaptive processing for noise cancelation in the speech signals. Subband processing employs the multirate signal processing. The polyphase based subband adaptive implementation finds better results in term of MMSE, PSNR and processing time; also the synthesis filter bank is works on the lower data rate which reduces the computational Burdon as compare to the direct implementation of Subband adaptive filter. The normalized least mean squares (NLMS) algorithm is a class of adaptive filter used.
\end{abstract}

Keywords: MMSE, NLMS, noise cancellation, PSNR, Polyphase based implementation.

\section{Introduction}

In practice the received speech signal contains some amount of noise component along with the information. The noise may be occurs due to coding of transmitted waveform (quantization noise) or an additive noise from background. Therefore for proper listing of this sound the noise need to be removed or suppressed form received noisy signal .The designing of such filter which removes or suppress noise required the signal and the noise be stationary that the statistics of both signals be known a priori. In practice, these conditions are rarely met. Various signal processing techniques have been proposed over the years for noise reduction in the signals. There are two different approaches for electrical noise reduction. The first approach is passive electrical noise reduction techniques, such as those applied in hearing aids, cochlear implants, etc. where the signal and ambient noise are recorded using a microphone, noise reduction techniques such as spectral subtraction, the LMS algorithm, etc. are applied and the listener hears only the clean signal. One of the important assumptions of this technique is that the listener is acoustically isolated from the environment. This assumption is however not valid in a large particularly those number of situations where the ambient noise has very large amplitude. In such situations, the second approach of Active Noise Cancellation (ANC) is applicable. ANC refers to an electromechanical or electroacoustic technique of cancelling acoustic various signal processing techniques have been proposed over the years for noise reduction in the signals. There are two different approaches for electrical noise reduction. The first approach is passive electrical noise reduction techniques, such as those applied in hearing aids, cochlear implants, etc. where the signal and ambient noise are recorded using a microphone, noise reduction techniques such as spectral subtraction, the LMS algorithm, etc. are applied and the listener hears only the clean signal [1]. One of the important assumptions of this technique is that the listener is acoustically isolated from the environment. This assumption is however not valid in a large particularly those number of situations where the ambient noise has very large amplitude. In such situations, the second approach of Active Noise Cancellation (ANC) is applicable. ANC refers to an electromechanical or electroacoustic technique of cancelling acoustic Disturbance to yield a quieter environment. The basic principle of ANC is to introduce a cancelling "antinoise" signal that has the same amplitude but the exact opposite phase, thus resulting in an attenuated residual noise signal. ANC has been used in a number of applications such as hearing protectors, headsets, etc. The traditional wideband ANC algorithms work best in the lower frequency bands and their performance deteriorates rapidly as the bandwidth and the center frequency of the noise increases. Most noise sources tend to be broadband in nature and while a large portion of the energy is concentrated in the lower frequencies, they also tend to have significant high frequency components. Further, as the ANC system is combined with other communication and sound systems, it is necessary to have a frequency dependent noise cancellation system to avoid adversely affecting the desired signal.

\section{Noise Cancellation}

The Noise Cancellation is a variation of optimal filtering that involves producing an estimate of the noise by filtering the reference input and then subtracting this noise estimate from the primary input containing 
both signal and noise. It makes use of an auxiliary or reference input which contains a correlated estimate of the noise to be cancelled. The reference can be obtained by placing one or more Sensors in the noise field where the signal is absent or its strength is weak enough. Subtracting noise from a received signal involves the risk of distorting the signal and if done improperly, it may lead to an increase in the noise level. This requires that the noise estimate $\mathrm{n}^{\wedge}$ should be an exact replica of $\mathrm{n}$. If it were possible to know the relationship between $\mathrm{n}$ and $\mathrm{n}$, or the characteristics of the channels transmitting noise from the noise source to the primary and reference inputs are known, it would be possible to make $\mathrm{n}^{\wedge}$ a close estimate of $\mathrm{n}$ by designing a fixed filter. However, since the characteristics of the transmission paths are not known and are unpredictable, filtering and subtraction are controlled by an adaptive process. Hence an adaptive filter is used that is capable of adjusting its impulse response to minimize an error signal, which is dependent on the filter output.

\section{Noise Cancellation Using Adaptive Filtering}

A basic concept of Adaptive Noise Canceller (ANC) removes or suppresses the noise from a signal using adaptive filters. Adaptive filters are digital filters with an impulse response, or transfer-function that can be adjusted or changed over time to match desired system characteristics [2]; they require little or no a priori knowledge of the signal and noise characteristics. (If the signal is narrowband and noise broadband, which is usually the case, or vice versa, no a priori information is needed; otherwise they require a signal (desired response) that is correlated in some sense to the signal to be estimated, Moreover adaptive filters. Have the capability of adaptively tracking the signal under non-stationary conditions.

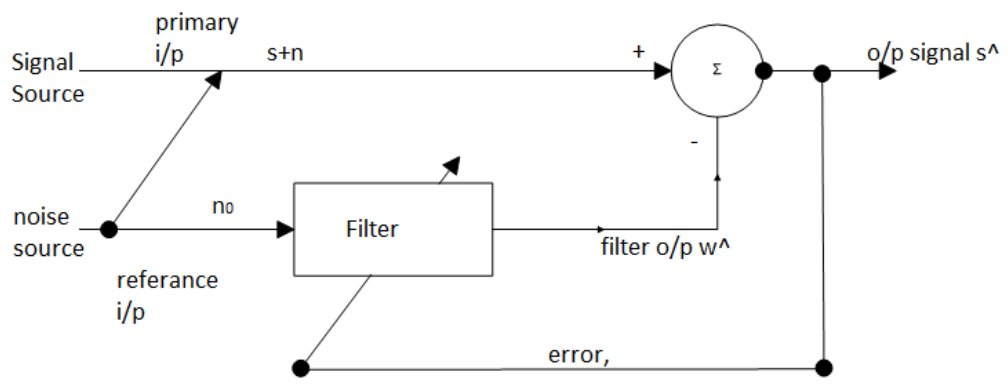

Fig.1 Adaptive Noise Canceller

The error signal to be used depends on the application. The criteria to be used may be the minimization of the mean square error, the temporal average of the least squares error etc. , Different algorithms are used for each of the minimization criteria e.g. the Least Mean Squares (LMS) algorithm, the Recursive Least Squares (RLS) algorithm etc. In the case of adaptive noise cancellation, we use the minimum mean-square error criterion. Examples of the adaptive filters are the Wiener filter, Recursive-Least Square (RLS) algorithm, and the Kalman filters were proposed to achieve the best performance. Among them Least Mean Square (LMS) algorithm is most commonly used because of its robustness and simplicity, However the LMS algorithm suffers from significantly degraded performance for colored interfering signals due to the eigenvalue spread of the autocorrelation matrix of the input signal. In addition, as the length of the adaptive filter increases, the computational complexity increases. This can be a serious problem in acoustic applications such as echo and noise cancellation, where long adaptive filters are required to model the response of the noise path. This issue is of great importance in the hands-free application where processing power is kept low. An alternative approach to reduce the computational complexity of long adaptive FIR filters is to incorporate block updating strategies and frequency domain adaptive filtering. These techniques reduce the computational complexity, because the filter output and the adaptive weights are computed only after a large block of data has been accumulated. However, the application of such approaches introduces degradation in the performance, including a substantial signal path delay corresponding to one block length, as well as a reduction in the stable range of the algorithm step size. Therefore for nonstationary signals, the tracking performance of the block algorithms generally becomes worse. As far as speed of convergence is concerned, it has been suggested to use the Recursive Least Square (RLS) algorithm to speed up the adaptive process. The convergence rate of the RLS algorithm is independent of the eigenvalue spread. Unfortunately, the drawbacks that is associated with RLS algorithm including its $\mathrm{O}(\mathrm{N} 2)$ computational requirements, which are still too high for many applications, where high speed is required, or when a large number of inexpensive units must be built. The Affine Projection Algorithm (APA) shows a better convergence behavior, but the computational complexity increases with the projection order in relation to LMS, where Projection denotes the order of the APA. As a result, adaptive filtering using subband processing becomes an attractive option for many adaptive systems to reduce these problems [4].

Multirate DSP consists of: 
1) Decimation: It is a process to decrease the sampling rate.

"Down sampling" is a process of removing some samples, without the low pass filtering. A signal is down sampled only when it is "oversampled"(i.e. sampling rate $>$ Nyquist rate). This combined operation of filtering and down sampling is called "Decimation". To down sample by a factor of M, we must keep every Mth sample as it is and remove the (M-1) samples in between.

2) Subband Processing.

Adaptive filter using the NLMS algorithm is applied in each band.

3) Interpolation: It is a process to increase the sampling rate.

"Up sampling" is the process of inserting zero valued samples between original samples to increase the sampling rate.

\section{Subband Adaptive Filtering in a Noise Cancellation Scenario}

Subband adaptive filtering belongs to two fields of digital signal processing, namely, adaptive filtering and multirate signal processing. The basic idea of SAF is to use a set of parallel filters to divide the wideband signal input of the adaptive filter into narrower subband signals, each subband serving as an input to an independent adaptive filter. Subband decomposition greatly reduces the adaptive filter update rate through parallel processing of shorter filters. Furthermore, subband signals are usually downsampled in a multirate system. A multirate DSP system uses multiple sampling rates within the system. Whenever a signal at one rate has to be used by a system that expects a different rate, the rate has to be increased or decreased, and some processing is required to doso. Therefore "Multirate DSP" refers to the art or science of changing sampling rates. "Resampling" means combining interpolation and decimation to change the sampling rate by a rational factor. Resampling is done to interface two systems with different sampling rates [5].

Multirate DSP consists of: 1 . Decimation: It is a process to decrease the sampling rate.

2. Interpolation: It is a process to increase the sampling rate.

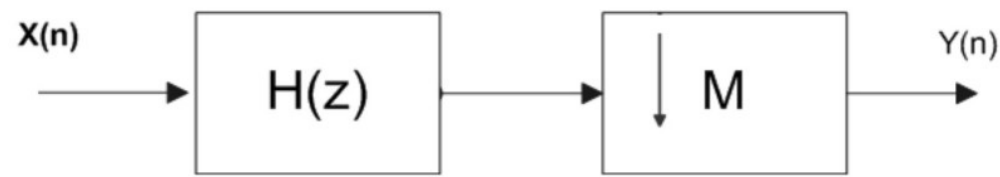

Fig.2. Block diagram of a decimator

"Down sampling" is a process of removing some samples, without the low pass filtering. A signal is down sampled only when it is "oversampled"(i.e. sampling rate $>$ Nyquist rate). This combined operation of filtering and down sampling is called "Decimation". To down sample by a factor of M, we must keep every Mth sample as it is and remove the (M-1) samples in between.

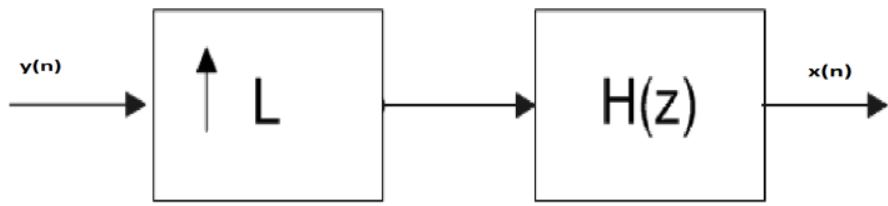

Fig.3. Block diagram of a decimator

"Up sampling" is the process of inserting zero valued samples between original samples to increase the sampling rate. (This is called "zero-stuffing"). "Interpolation" is the process of upsampling followed by filtering (to remove the undesired spectral images.) The result is a signal sampled at a higher rate. The interpolation factor $(\mathrm{L})$ is the ratio of the output rate to the input rate. This leads to a whitening of the input signals and therefore an improved convergence behavior of the adaptive filter system is expected. The subband decomposition is aimed to reduce the update rate, and the length of the adaptive filters, hopefully, resulting in a much lower computational complexity [4]. The conventional noise cancellation model is extended to a subband configuration by the insertion of sets of analysis and synthesis filters in signal paths. Both input signals $\mathrm{s}$ and $\mathrm{n}$ are now fed into identical M-band analysis filter banks $\mathrm{H}(\mathrm{z})$, with $\mathrm{n}^{\wedge}$ being a filtered version of $\mathrm{n}$ by an unknown system $\mathrm{P}(\mathrm{z})$. Here, $\mathrm{P}(\mathrm{z})$ represents the acoustic noise path, $\mathrm{n}$ being correlated with and uncorrelated with $\mathrm{s}$. The ultimate goal is to suppress $\mathrm{n}^{\wedge}$ at the output $\mathrm{s}$ and to retain the non-distorted version of $\mathrm{s}$. The update equation of the adaptive filter using the LMS algorithm is given by the following set of equation. Depending on the decimation rate used for analysis and synthesis filter bank the subband structure is divide as, 


\section{A. Critically Sampled}

In critically sampled the decimation factor is equal to the number of subband.

\section{B. Oversampled Structures}

In Oversampled structure the decimation factor is greater than the number of subbands.

\section{NLMS Adaptive Algorithm}

The main drawback of the "pure" LMS algorithm is that it is sensitive to the scaling of its input $\mathrm{x}(\mathrm{n})$. This makes it very hard (if not impossible) to choose a learning rate $\mu$ that guarantees stability of the algorithm (Haykin 2002). The Normalised least mean squares filter (NLMS) is a variant of the LMS algorithm that solves this problem by normalising with the power of the input. The NLMS algorithm can be summarised as:

Parameter: $\mathrm{p}=$ filter order

$$
\begin{aligned}
& \quad \mu=\text { step size } \\
& \text { Initialization: } \hat{h}(0)=\operatorname{zero}(p) \\
& \text { Computation: For } n=0,1,2, \ldots \\
& \boldsymbol{x}(\boldsymbol{n})=[\boldsymbol{x}(\boldsymbol{n}), \boldsymbol{x}(\boldsymbol{n}-\mathbf{1}), \ldots, \boldsymbol{x}(\boldsymbol{n}-\boldsymbol{p}+\mathbf{1})]^{T}
\end{aligned}
$$

Error equation,

$$
e(n)=d(n)-\widehat{h}^{H}(n) x(n)
$$

Weights update equation for standard LMS algorithm

$\widehat{w}(n+1)=\widehat{w}(n)+\mu u(n) e^{*}(n)$

Weights update equation for standard NLMS algorithm

$\widehat{w}(n+1)=\widehat{w}(n)+\frac{1}{\|\boldsymbol{u}(n)\|^{2}} u(n) e^{*}(n)$

\section{A. Direct Implementation}

\section{Filter Banks}

In the Analysis filter bank a signal is being separated into its low frequencies and high frequencies. For that the LPF and HPF is used, their corresponding impulse responses are H0 (n) and H1 (n) respectively, with a cutoff frequency at pi/2, as. The sub-band signals V0 (n) and V (n) are then down-sampled by a factor of 2. Adaptive processing is done in both subband separately. The perfect reconstruction process requires four filters, two lowpass filters (H0 and $\mathrm{G} 0)$ and two highpass filters (H1 and G1) [7]. In addition, it requires a downsampler and upsampler between the two lowpass and between the two highpass filters. The combination of Decimation filter and low pass filter makes together analysis filter bank similarly the synthesis filter bank consist of interpolation and filters. In this way, the aliasing in the analysis/synthesis bank is easily eliminated. The distortion transfer function $\mathrm{T}(\mathrm{z})$ is expressible in terms of the lowpass filter $\mathrm{H} 0 \mathrm{(z}$ ).

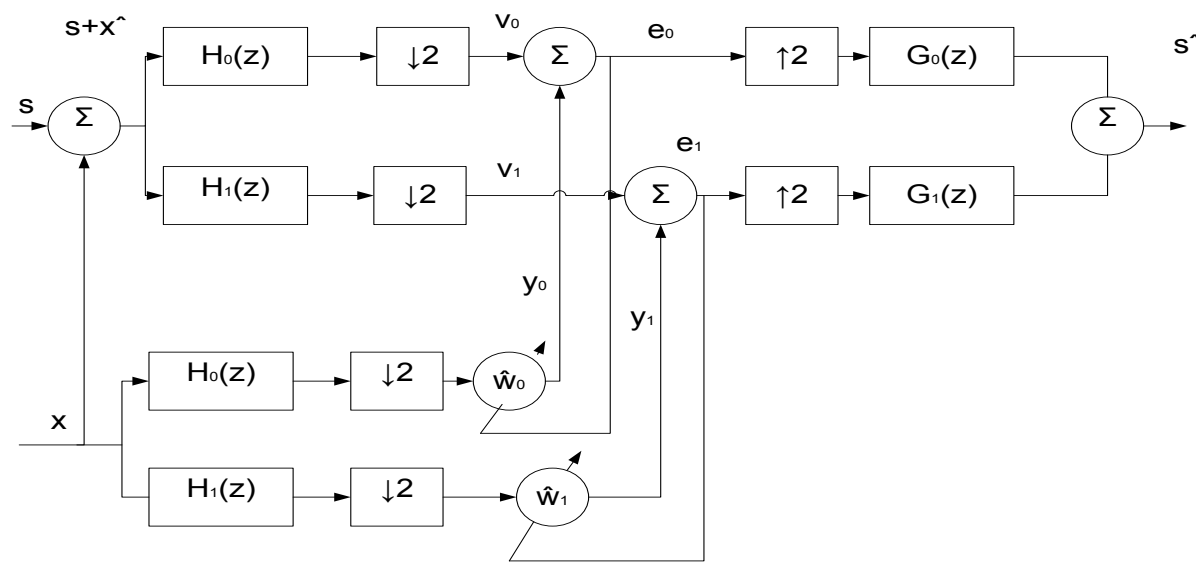

Figure.4. Direct implementation for Subband adaptive filtering

\section{B. Proposed Implementation}

In direct implementation the filtering is applied to all original signal samples, even though only every $\mathrm{M}^{\text {th }} \quad$ filtering output is retained finally. Even if $\mathrm{H}(\mathrm{z})$ operates only for time instants multiple of $\mathrm{M}$ and idle 
otherwise, all multipliers/adders have to produce results within one step of time. This additional Computational burden can be reduced by the use of noble identities of the decimation and filter. Here $\downarrow$ 2(Decimation Filter) can be moved before H (z) by use of noble identities [6] [8].This is called Efficient Structure for Decimation Filter using Type-1 polyphase representation.

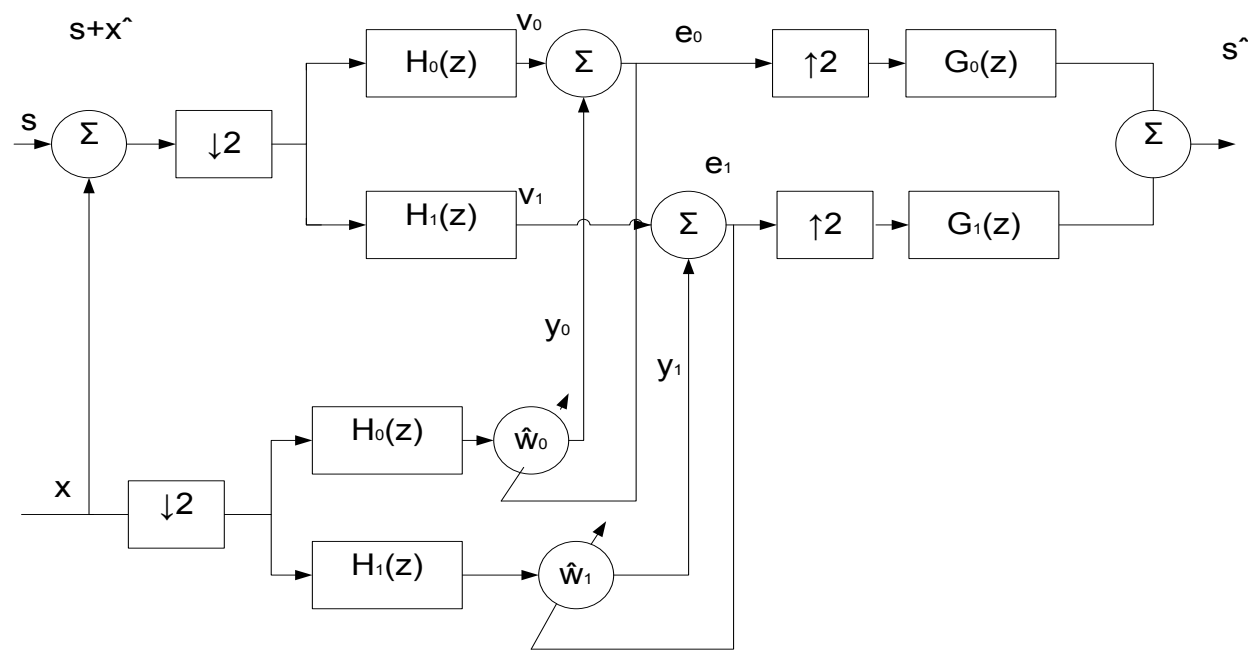

Figure.5. Efficient Structure for Subband adaptive filtering

\section{A. Minimum Mean Square Error (MMSE):}

\section{Performance Parameter}

Let us assume that a random variable $\mathrm{Y}$ and it is like to estimate its value, knowing only its probability density function. We will then broaden the discussion to estimation when we have a observation of another random variable $X$, together with the joint probability density function of $X$ and $Y$. To quantify the difference between the values implied by an estimator and the true values of the quantity being estimated MSE is defined as,

$M S E=\frac{1}{n} \sum_{n}^{n}\left(\widehat{Y}_{\imath}-Y_{i}\right)$

Based only on knowledge of the PDF of Y, we wish to obtain an estimate of Y - which we denote as $\mathrm{y}-\mathrm{so}$ as to minimize the mean square error between the actual outcome of the experiment and our estimate $\mathrm{y}$. Specifically, we choose y to minimize. The associated error-the actual MMSE-is found evaluating the expression 2 . The MMSE is just the variance of Y namely $\sigma_{y}^{2}$.

$\min E\left[(Y-\hat{y})^{2}\right]=E\left[(Y-E[Y])^{2}\right]=\sigma_{y}^{2}$

\section{B. Peak Signal to Noise Ratio (PSNR):}

Peak signal-to-noise ratio, often abbreviated PSNR, is the ratio between the maximum possible power of a signal and the power of corrupting noise . Because many signals have a very wide dynamic range, PSNR is usually expressed in terms of the logarithmic decibel scale. Although a higher PSNR generally indicates that the reconstruction is of higher quality, in some cases it may not. PSNR is most easily defined via the mean squared error (MSE).

The PSNR (in $\mathrm{dB}$ ) is defined as:

$P S N R=10 \cdot \log _{10}\left(\frac{M A X^{2}}{M S E}\right)$ 
I. EXPERIMENTAL RESULTS

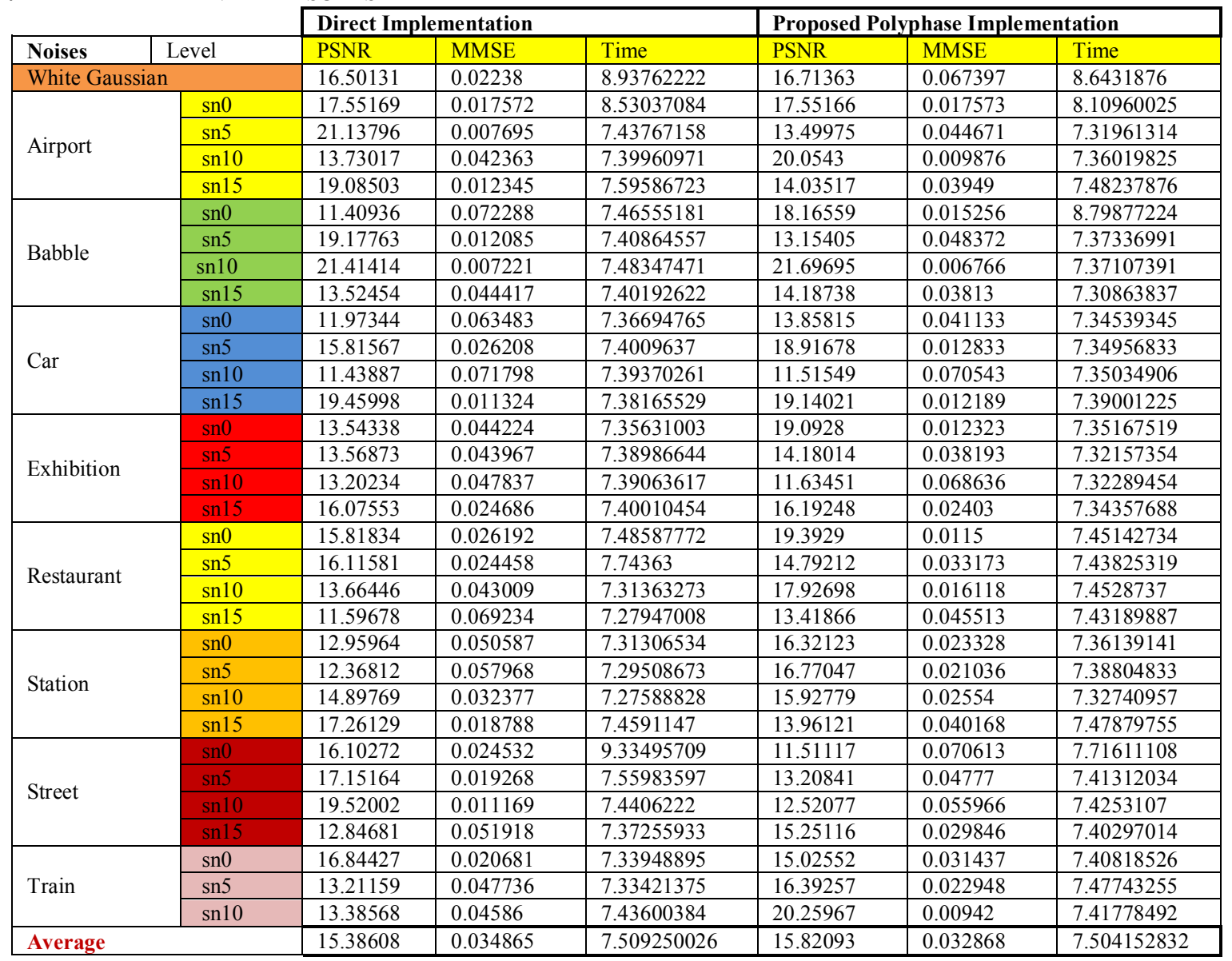

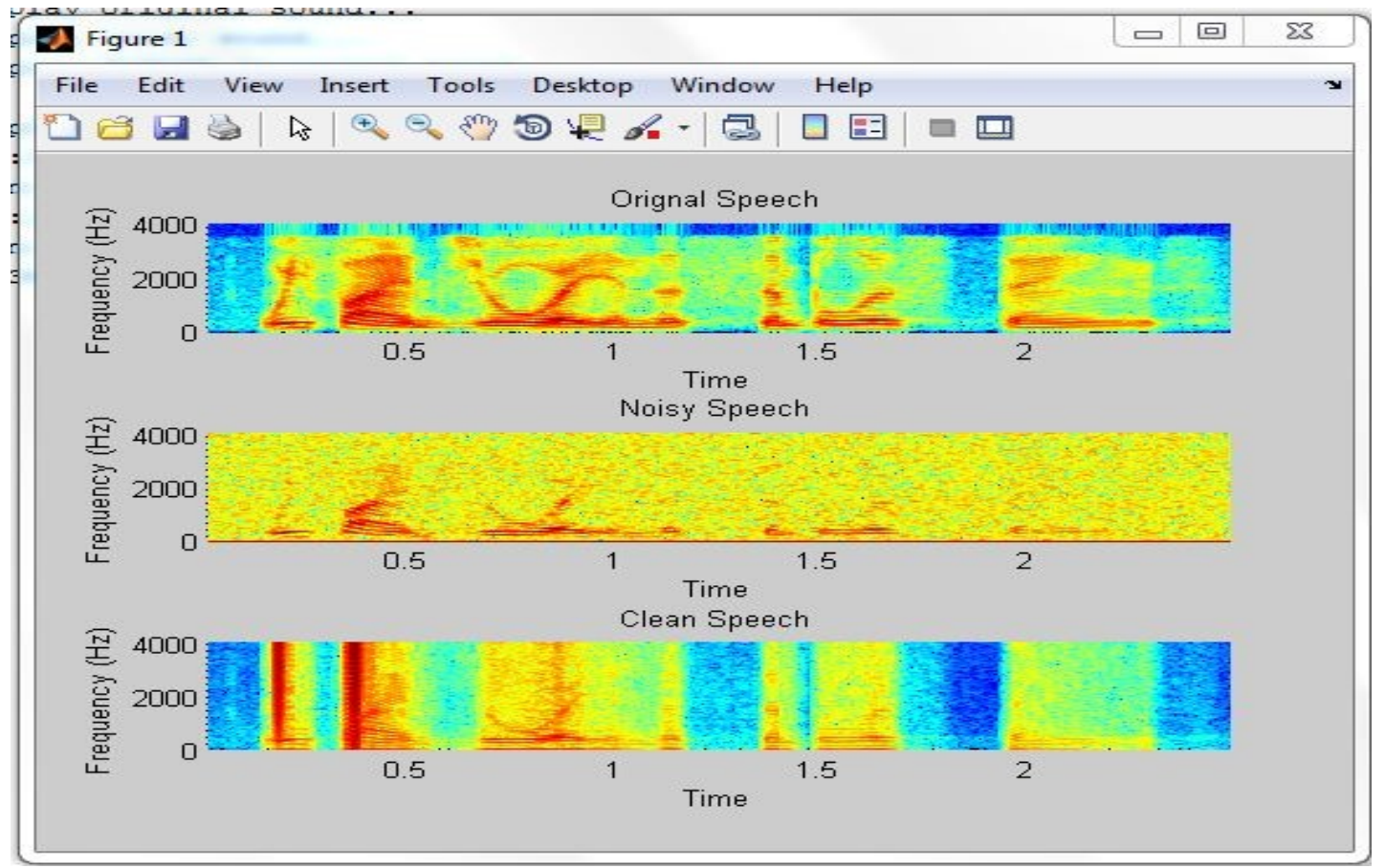

Fig.6.Spectrogram for direct implementation 


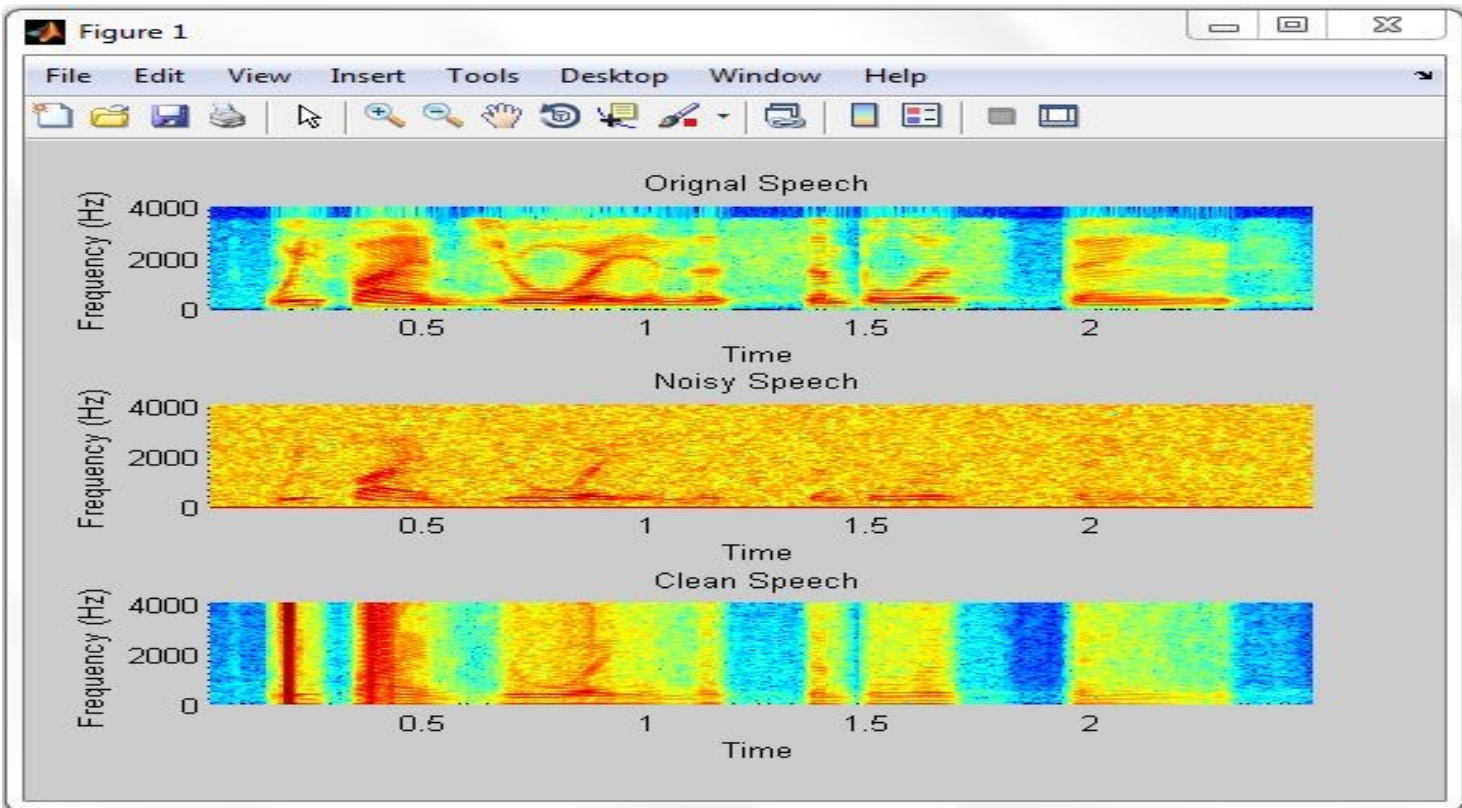

Fig.7.Spectrogram for Proposed polyphased implementation

VIII. Conclusion

Subband adaptive filtering is done for noise cancellation in the speech .The experimental results shows that when NLMS algorithm as adaptive filter for noise cancellation in the subband with noble identities is used; it fined better results over convention approach i.e. direct implementation. Also the analysis filter bank is working at lower data rate it saves the computational cost by $\mathrm{M}$ factor (Decimation factor).

\section{References}

[1]. B. Widrow, (1975) Adaptive Noise Cancelling: Principles and Applications; Proc. IEEE, vol. 63, pp.1692-1716.

[2]. Simon Haykin(1996)Adaptive Filter Theory 3rd edition; Prentice Hall, Englewood Cliffs, New Jersey.

[3]. Ali O. Abid Noor(2011) Adaptive Filtering Using Subband Processing: Application to Background Noise Cancellation; ISBN 978953-307-158-9.

[4]. Noor, A.O.A., S.A. Samad and A. Hussain,(2011) Adaptive Filtering using Subband Processing: Application to Background noise Cancellation. In "Adaptive Filtering"; ITech: Vienna, Austria,pp: 109-134.

[5]. "Digital Signal Processing-A Practical Approach”, Emmanuel Ifeachor,Barrie W.Jervis .ISBN 978-81-317-0824-8,Person

[6]. "Multirate Filtering for Digital Signal Processing-MATLAB Applications",Ljilana Milic,ISBN 978-1-60566-179-7,Premier Reference Source

[7]. Ali O. Abid Noor, Salina Abdul Samad and Aini Hussain"A Review of Advances in Subband Adaptive Filtering" World Applied Sciences Journal 21 (1): 113-124, 2013 ISSN 1818-4952

[8]. Noble Identities, Filters,Gerald Schuller, TU-Ilmenau, https://ccrma.stanford.edu/ jos/sasp/Multirate_Noble_Identities.html

[9]. Riitta Niemist and Ioan Tabu, (2001), Signal Adaptive Subband Decomposition for Adaptive Noise Cancellation1, European Conference on Circuit Theory and Design.

[10]. Ali A. Milani, M. S. Panahi, (2009) A New Delayless Subband Adaptive Filtering, Algorithm for Active Noise Control Systems;IEEE Transactions on Speech, And language Processing, VOL. 17, No. 5.

[11]. NOIZEUS: A noisy speech corpus http://www.utdallas.edu/ loizou/speech/noizeus/, Feb, 2013 